\title{
USING MICROSOFT TEAMS TO SUPPORT COLLABORATIVE KNOWLEDGE BUILDING IN THE CONTEXT OF SUSTAINABILITY ASSESSMENT
}

\author{
Ralph Buchal ${ }^{1}$ and Emmanuel Songsore ${ }^{2}$ \\ ${ }^{1}$ Western University, ${ }^{2}$ Wilfred Laurier University \\ rbuchal@uwo.ca
}

\begin{abstract}
The current study investigates students' use of Microsoft Teams as a collaborative knowledge building platform for a group sustainability assessment project. Ashby's sustainability assessment method was used to provide scaffolding. Surveys $(n=16)$ were administered to assess the nature of student collaboration, including students' experiences using collaboration tools in the past, the activities students engaged in while working on the group project in MS Teams, self-assessment of collaborative abilities, comfort with giving, receiving and sharing comments and feedback, assessment of the effectiveness of Ashby's sustainability assessment method in developing these abilities, and their overall assessment of MS Teams as a collaborative knowledge building tool. Students rated their collaborative abilities to be good to excellent and felt that the project was effective in developing those abilities. They are comfortable providing and receiving feedback and sharing their contributions openly. They found MS Teams to be extremely useful, and better than alternative platforms for key tasks including messaging, file sharing and collaborative authoring.
\end{abstract}

Keywords: Sustainability, collaborative knowledge building, computer supported collaborative learning, Microsoft Teams

\section{INTRODUCTION}

Collaborative knowledge building is an established theory and framework for learning [1], [2], [3]. Stahl has proposed a collaborative knowledge building model (Figure 1) that explicitly identifies key activities including: articulating personal perspectives and viewpoints; comparing and integrating multiple perspectives; identifying and reconciling contradictions; filling gaps; clarifying and negotiating meanings; linking new knowledge into existing personal and group knowledge structures; modifying beliefs; and reflecting on learning [1], [4], [5]. Computer-based collaboration platforms can enhance collaborative knowledge building by supporting these key collaborative activities [1], [3], [6], [7], [8], [9].

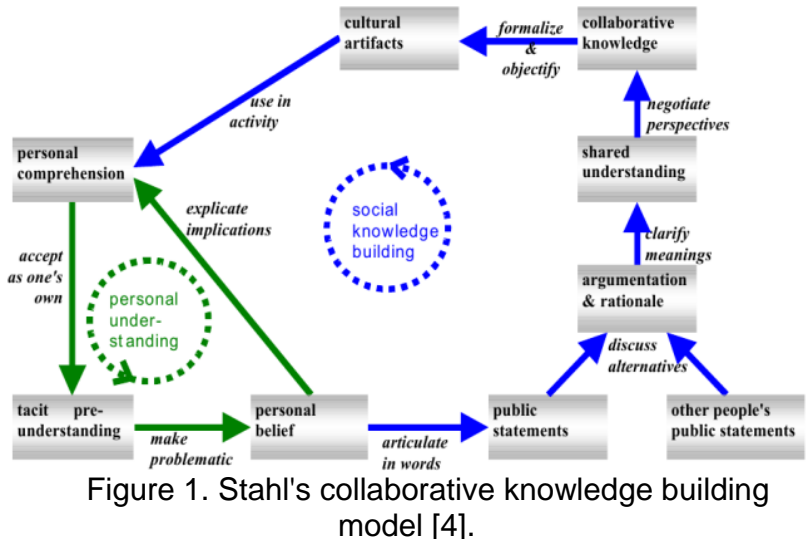

Only a few researchers have analyzed the adequacy of existing collaboration tools (e.g. [4], [8], [9]), and many available tools lack affordances to effectively support rich collaborative activities. Also, many studies observe how students use collaboration tools naturally, without any intervention or guidance (e.g. [10]). There is evidence that students do not engage in rich collaborative behaviours naturally [6], [9], [11] and many researchers now agree that intentional training and on going support from instructors are key ingredients for rich and successful collaboration among students [6], and that supports in the form of scripts, training and guidance can be effective in developing collaborative practices [9], [12].

\section{IDENTIFICATION AND EVALUATION OF COLLABORATION TOOLS}

The first author has reviewed and evaluated available collaboration tools, based on the affordances required to support rich collaborative activities as described by Stahl and others (redacted). No integrated tool was found that meets all the requirements, but among available tools Microsoft SharePoint was found to be one of the most capable. The first author has many years of experience with SharePoint (redacted). A recent study using SharePoint as a platform for collaborative knowledge building showed that students found it easy to use and useful for supporting 
collaborative projects, but that it still has a number of shortcomings [9]. Microsoft has recently introduced the Teams collaboration app as part of Office 365 [13]. Microsoft (MS) Teams extends the functionality of Microsoft SharePoint, with a simplified user interface and the addition of a group chat capability. It is available as an app for iOS and Android devices, as well as running in a browser or app on a Windows or Mac computer. It is part of Office 365, and is included in the educational license of Office 365 available at many universities.

\section{SUSTAINABILITY ASSESSMENT METHOD}

The sustainability assessment method developed by Ashby et al. [14], [15] provides a systematic framework for dealing with complex, multidisciplinary problems, and promotes a systems-thinking approach (Figure 2). It is also an excellent framework to provide scaffolding for collaborative knowledge building. The method is used to assess the sustainability of a proposed solution. The method consists of five stages: problem definition, stakeholder analysis, fact-finding, synthesis and evaluation, and reflection. The problem definition stage defines the objectives, scope and time scale of a proposed solution to be assessed. Stakeholder analysis identifies the stakeholders and their concerns. The fact-finding stage gathers factual information in several categories: materials, energy, environment, legislation and regulations, economics and society. This ensures that all perspectives are considered. The synthesis and evaluation stage uses the facts along with argumentation, judgement and reasoning to assess the positive and negative impacts on environmental, economic and social sustainability. The reflection stage reflects on the positive and negative impacts and reaches conclusions about whether the objective is achievable, and whether the benefits outweigh the negative impacts. Reflection also suggests new or different ways to achieve the objective.

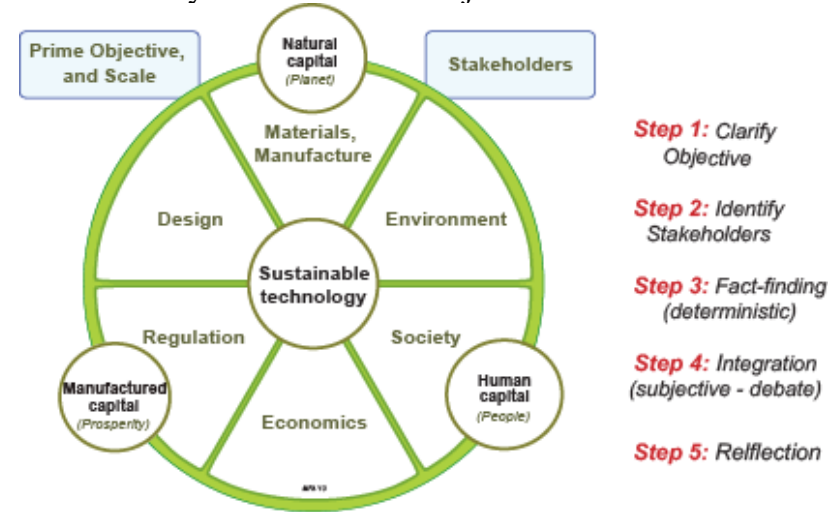

Figure 2. Ashby's Sustainability Assessment Framework [14].

Ashby's method is intended to be used in conjunction with the CES Edupack material selection software [16]. CES Edupack includes searchable databases of nations of the world, legislation and regulations, energy storage systems, low carbon power systems, and nuclear energy systems. The nations of the world database includes a variety of information about every nation in the world. The information is grouped into several categories: demographic information, education, human rights and governance, economy and development, health, energy, carbon and environment, and military expenditures. The low carbon power database contains a description, resource intensity, operational parameters, and status of most if not all power generation technologies. These technologies include: conventional fossil fuels, fuel cells, geothermal, hydro, nuclear, biomass, solar, wind, wave, and tidal.

The sustainability assessment method is very effective in evaluating the feasibility of a proposed solution or articulation, but it does not help in generating alternative solutions based on a problem or need. Ashby and his colleagues show several worked examples of the assessment of particular solutions, but he does not extend this to a comparison and ranking of multiple alternatives. However, this extension is straightforward.

In the domain of sustainability, there are many known articulations or solutions, and each can be assessed using this method. A comparison of alternatives would involve the comparative assessment of several candidates. For example, the method could be used to assess wind energy and solar energy as two alternative solutions, and the results could be compared.

\section{DESCRIPTION OF COURSE PROJECT}

A sustainability assessment project was introduced in Fall 2017 in a third-year materials selection course in the mechatronics systems engineering program at (redacted) University. The overall project objective was to "reduce carbon emissions enough to avoid the worst consequences of climate change. "Students worked in groups to carry out a sustainability assessment of a solution of their choice. In some cases, they chose solutions to other environmental issues. Solutions that they assessed included: clean meat, biodegradable plastics, geothermal power, wave power, microgrid power, nuclear power, conflict minerals in electronics, sustainable freight trains, delivery drones, sustainable clothing, afforestation, and Elon Musk's traffic tunnels. There were 17 teams of 3-5 students each.

\section{USING MICROSOFT TEAMS}

The teams collaborated online using Microsoft Teams [13]. Each Team was configured with "channels" corresponding to the stages of the sustainability assessment method: problem definition; stakeholder analysis; factfinding; synthesis; and conclusions and recommendations. Each channel was configured with a group chat conversation, a file document library, and a shared OneNote notebook. Setting up a team takes a couple of 
minutes. The instructor (Redacted) was a member of every team and participated in team conversations.

\section{METHODOLOGY}

Students were introduced to Ashby's sustainability assessment method, and a detailed guide was provided. Students were required to use MS Teams, and the instructor monitored student contributions and provided suggestions and feedback. A word document template was provided with sections corresponding to the stages of Ashby's method.

After the completion of the course, an online survey was administered to address several questions:

- What activities and strategies have students used in the past when working on group projects?

- What collaboration and communication modes do they use currently?

- How comfortable are students with giving and receiving constructive feedback?

- How comfortable are students with having their contributions visible to instructor and/or teaching assistant?

- How do students rate their collaborative abilities?

- How do students rate the effectiveness of the project in improving their collaborative abilities?

- How easy did students find MS Teams to learn and use?

- How effective did students find MS Teams in supporting collaborative activities?

- How frequently did students use various MS Teams affordances?

- How did students rate MS Teams in comparison to other online tools they have used?

\section{RESULTS}

In each of the following sections, the survey question is stated, followed by the survey results.

\subsection{Current Activities and Strategies}

Several research studies (e.g. [6], [9], [11]) have shown that students do not naturally engage in collaborative activities, and that when working on group projects they tend to divide the project into tasks that are assigned to individual members of the team. A survey question was developed to assess students' current collaborative activities.

Question: When you have worked on group projects in the past, how often did you and your group members engage in the following activities? a) Dividing the work into parts and assigning specific tasks to each member.

b) Working on assigned tasks independently and combining the parts at the end.

c) Presenting arguments to the group.

d) Sharing information from authoritative sources.

e) Negotiating to find a consensus.

f) Responding to arguments with alternative arguments.

g) Discussing particular perspectives or points of view.

h) Debating alternative perspectives or points of view.

i) Tracking individual progress on assigned tasks.

j) Collectively clarifying definitions and terminology.

k) Disagreeing with the perspective of colleagues.

l) Correcting the grammar of colleagues.

The results of this question $(n=16)$ are summarized in Figure 3

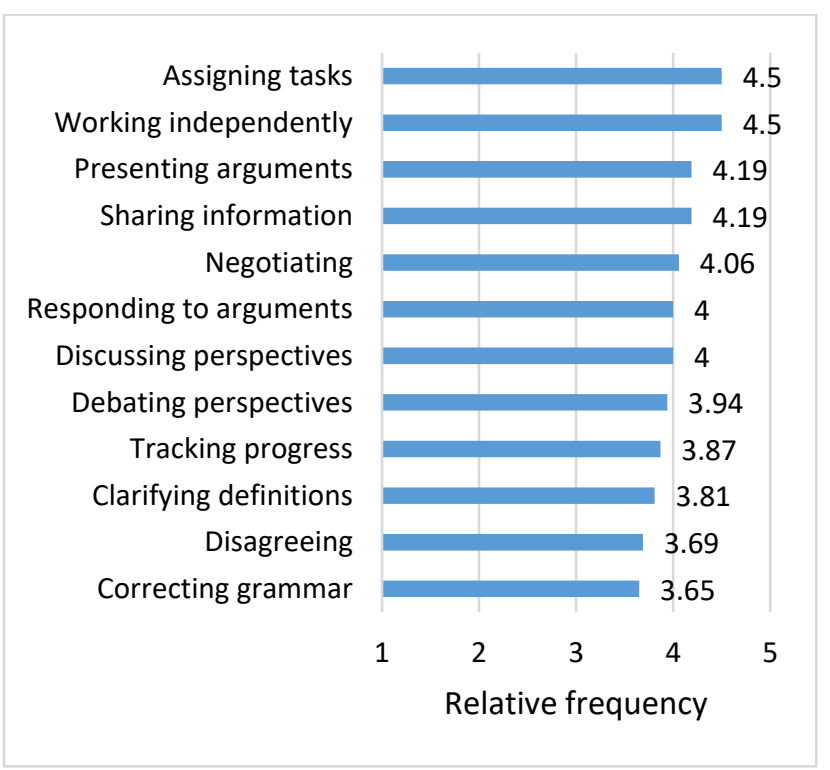

Figure 3. Frequency of group activities in previous projects $(n=16)$. 1-never, 2-rarely, 3-sometimes, 4-most of the time, 5-always.

Most student indicated that they normally divide a project into tasks and assign them to individuals, who work on their assigned tasks independently. This is consistent with other studies (e.g. [6]), and with empirical experience.

The least common activities include disagreeing with the perspectives of teammates, and correcting teammates' grammar. 


\subsection{Current Collaboration Modes}

Group work is a central feature of most engineering curricula, but few schools provide collaboration platforms to support group work. As "digital natives" it is commonly assumed that students will find and use tools on their own without specific guidance. This survey question is intended to identify the tools most commonly used by students.

Question: Indicate the how often you use different modes of collaboration and communication when working on group projects with your colleagues.

a) Collaborative authoring platforms (e.g. Google Docs).

b) File sharing.

c) Text messaging.

d) Face-to-face meetings.

e) Email.

f) Phone calls.

g) Videoconferencing.

The results of this question $(\mathrm{n}=16)$ are summarized in Figure 4.

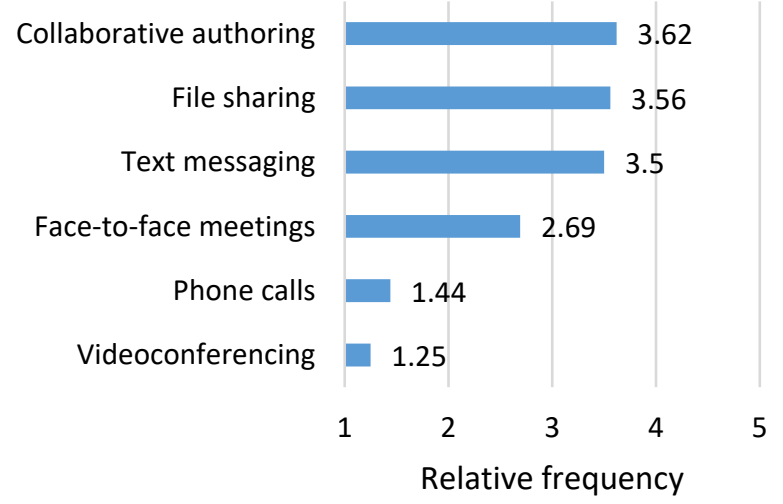

Figure 4. Use of collaboration modes $(n=16)$. 1-never, 2 - once a week, 3 - 2 to 3 times a week, 4 - 4 to 6 times a week, 5 - daily.

Most students indicated that the most common collaboration tools were group texting or group chat, file sharing, and collaborative authoring. Phone calls and email are rarely used. Two students reported using Facebook Messenger for group chats.

\subsection{Comfort Level}

It is possible that students do not engage in rich collaborative activities because they are uncomfortable providing or receiving criticism. This question probes their comfort level.

Question: How comfortable are you in providing constructive feedback or criticism of the ideas and viewpoints of other members of the team?
Question: How comfortable are you in receiving constructive feedback or criticism of the ideas and viewpoints of other members of the team?

The results of this question are shown in Figure 5.

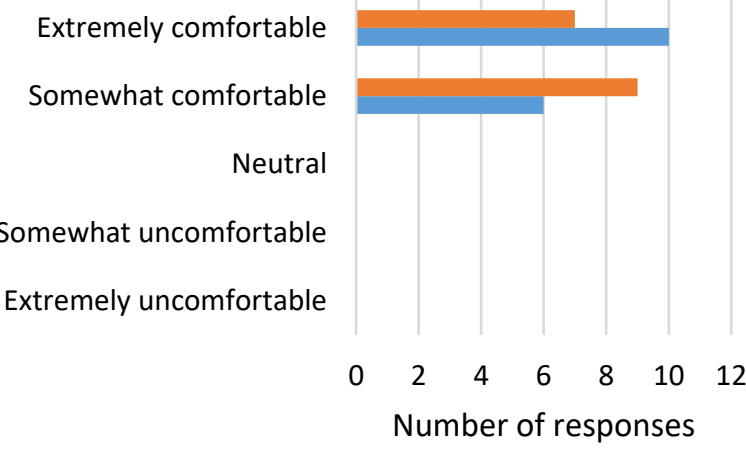

Providing feedback Receiving feedback

Figure 5. Student comfort providing and receiving constructive feedback and criticism $(n=16)$.

Student responses suggested that they were generally comfortable receiving constructive feedback but were less comfortable giving feedback. All respondents were at least somewhat comfortable giving and receiving constructive feedback. This suggests that requiring students to engage in these activities does not make them uncomfortable.

Students are not used to having professors and teaching assistants observing and participating in their team communications, and it is possible that this makes them uncomfortable.

Question: How comfortable are you in having your comments and contributions visible to all members of the team? The course instructor or TA?

The results of this question are shown in Figure 6 and Figure 7.

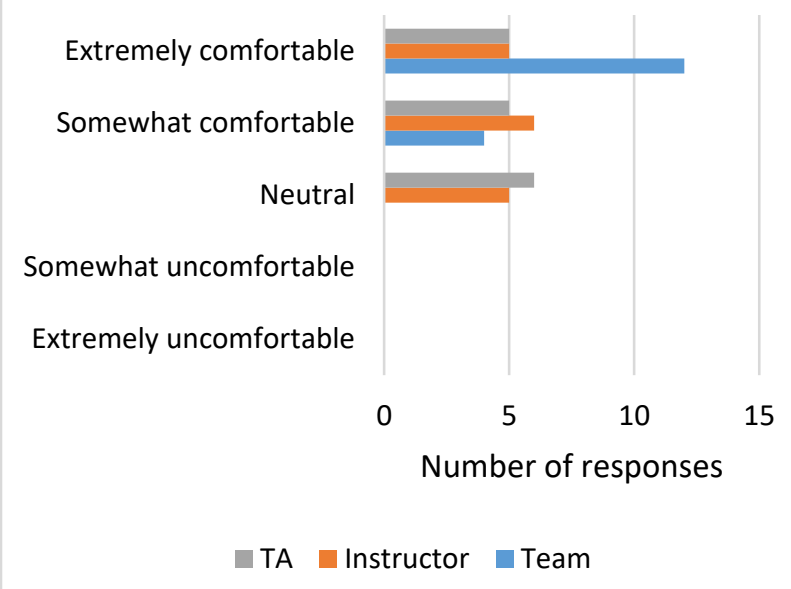

Figure 6. Student comfort sharing contributions with teammates, instructor and teaching assistant $(n=16)$. 


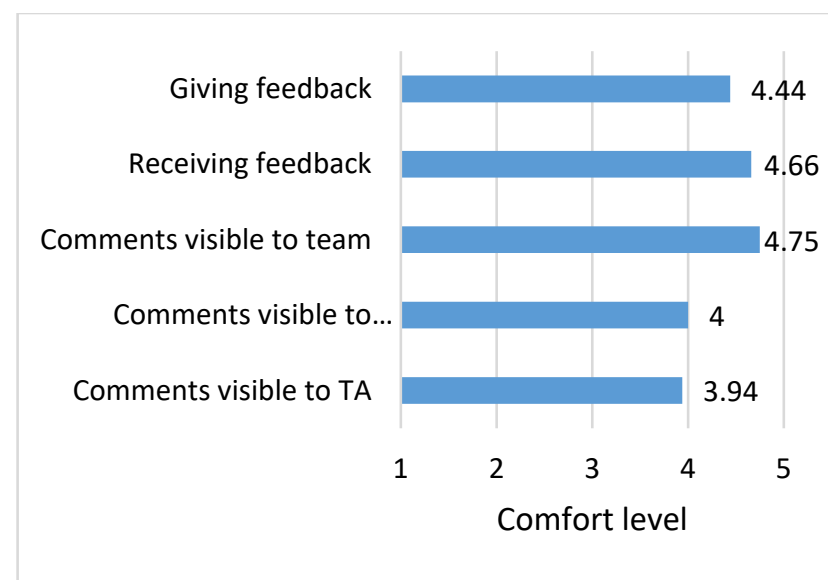

Figure 7. Student comfort giving feedback, receiving feedback, sharing with teammates, sharing with instructor, and sharing with TA $(n=16)$.

The majority reported being extremely comfortable with having their contributions and comments visible to the entire team. They were generally less comfortable having their project visible to the instructor and TA, but none were uncomfortable. Some respondents noted that having their team communications visible to the instructor or TA forced them to be more professional in their communications.

\subsection{Collaboration Abilities}

There is evidence from experience and research studies that students do not naturally develop collaborative abilities just by working in groups. Two questions were asked to probe students' self-assessment of their collaborative abilities, and the effectiveness of the project in developing those abilities.

Question A: Before the project, rate your ability in the following areas.

Question B: How effective was the project in improving your abilities in these areas?

a) Formulating coherent arguments based on evidence and sound reasoning.

b) Organizing knowledge obtained from multiple sources.

c) Acknowledging uncertainty and incomplete information when tackling a complex problem.

d) Negotiating with others to come to a consensus.

e) Incorporating alternative viewpoints or perspectives into your own thinking.

f) Recognizing the limitations of your personal knowledge.

g) Seeking information from authoritative sources.

h) Recognizing and appreciating the merits of alternative viewpoints or perspectives.

The results of this question are shown in Figure 8.

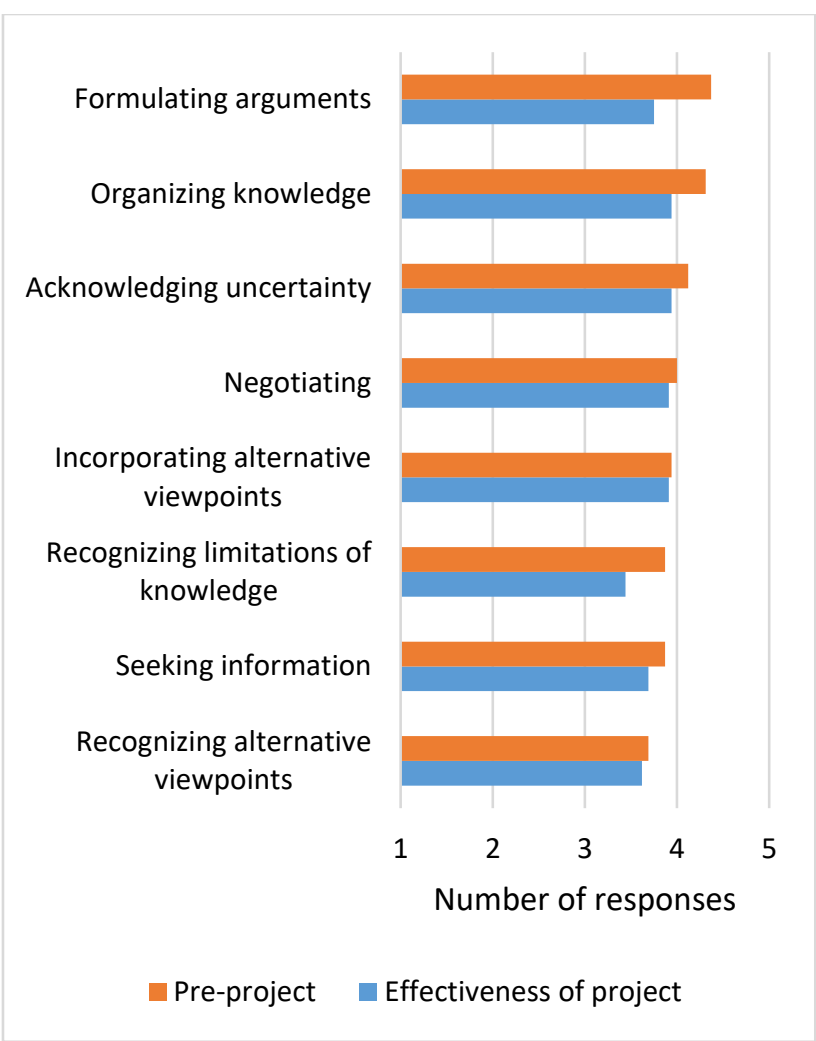

Figure 8. Pre-project abilities, and effectiveness of project in developing abilities $(n=16) .1$ - poor, 2 - fair, 3 good, 4 - very good, 5 - excellent.

Almost all respondents rated their pre-project collaboration abilities between good and excellent, and most agreed that the project was effective in improving these abilities. It is worth noting that these abilities also correspond to many models of critical thinking. It is possible that students overrate their abilities, as there was no strong evidence of some of these activities in the Microsoft Teams conversation transcripts and document comments.

\subsection{Effectiveness of Ashby's Method}

Many researchers agree that scaffolding and guidance are required to promote effective collaborative activities. This question probes the effectiveness of Ashby's method in providing guidance.

Question: How effective was Ashby's 5-step sustainability assessment method in supporting and guiding your collaborative project?

The results are shown in Figure 9. 


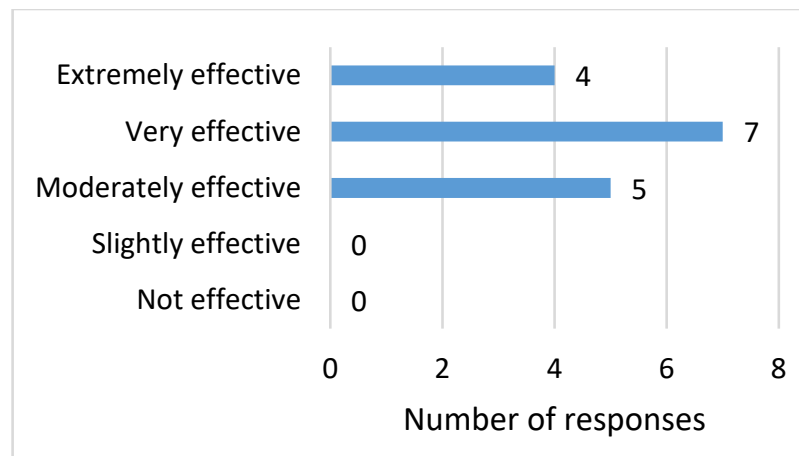

Figure 9. Distribution of ratings for overall effectiveness of Ashby's sustainability method $(n=16)$.

All students found Ashby's sustainability assessment method to be effective in providing guidance for their project.

Question: How effective was Ashby's 5-step method in supporting the following collaborative knowledge building activities?

a) Defining and clarifying the prime objective and scale of the proposed solution.

b) Reflecting on implications and forming a consensus about conclusions and recommendations.

c) Identifying the stakeholders and their concerns.

d) Identifying and collecting relevant facts from different areas (e.g. environment, regulation, etc.).

e) Integrating and synthesizing factual information, using judgment and reasoning, to assess impacts on natural, manufactured and human capitals.

The results are shown in Figure 10.

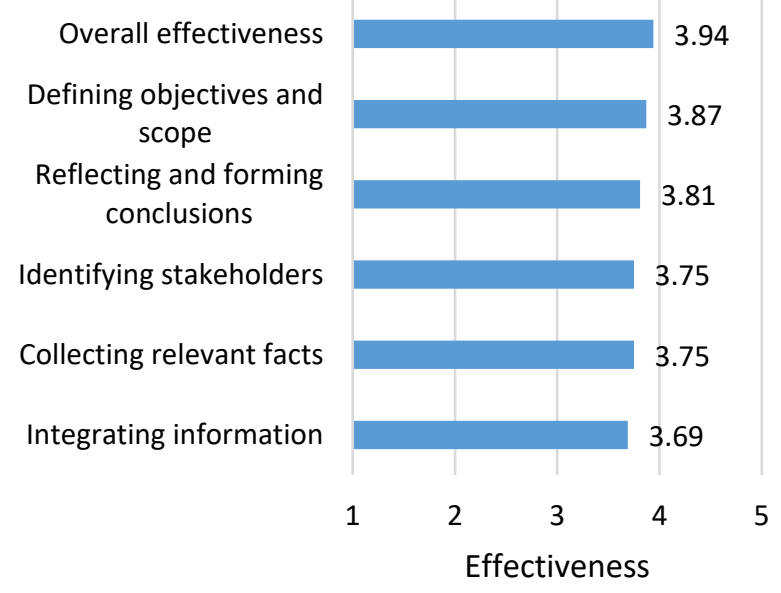

Figure 10. Student assessment of effectiveness of Ashby's sustainability assessment method $(n=16)$. 1 - not effective, 2 - slightly effective, 3 - moderately effective, 4 very effective, 5 - extremely effective.
The majority of students found Ashby's method to be moderately to extremely effective in supporting collaborative knowledge building activities.

\subsection{Assessment of Microsoft Teams}

Question: How easy was Microsoft Teams to learn and use?

The results are shown in Figure 11.

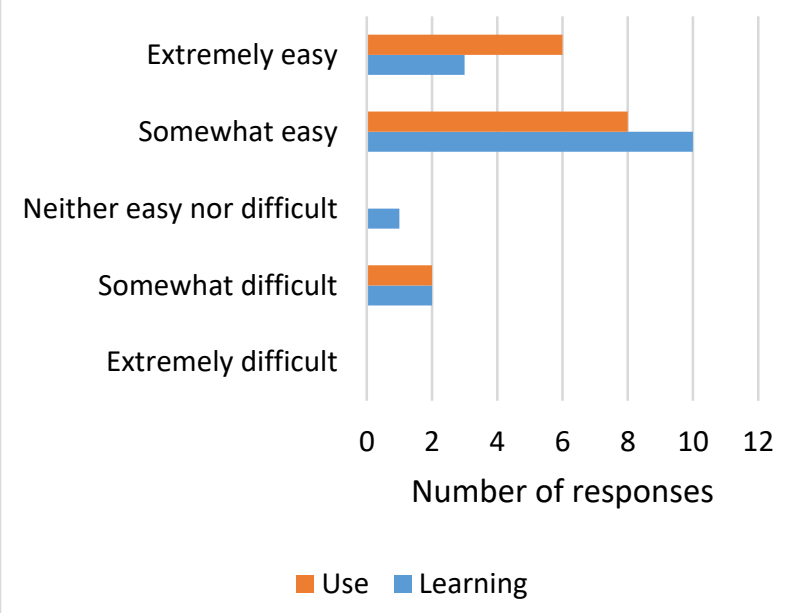

Figure 11. Student assessment of ease of learning and ease of use of Microsoft Teams $(n=16)$.

Question: How useful was Microsoft Teams in supporting your collaboration?

The results are shown in Figure 12.

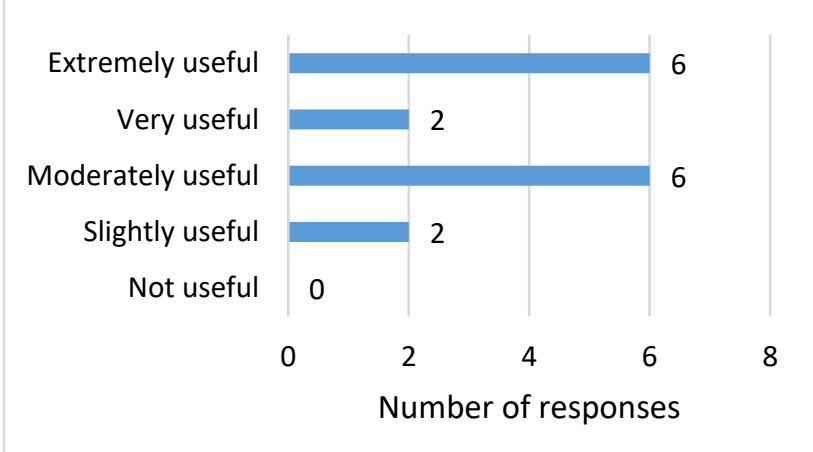

Figure 12. Student assessment of usefulness of Microsoft Teams $(n=16)$.

Most students found MS Teams easy to learn and use, but a minority found it somewhat difficult. Most students also found Teams to be useful in supporting collaboration with $37.5 \% \quad(n=16)$ finding it extremely useful. The majority $(81.25 \%, \mathrm{n}=16)$, found it better than other online tools they have used (e.g., google docs, Facebook, email, etc.). Some students noted the benefits of having a single integrated platform for communication, file sharing and collaborative authoring, with a single login account. 
Question: Compared to other online tools you have used, how effective was MS Teams in supporting your collaborative activities?

The results are shown in Figure 13.

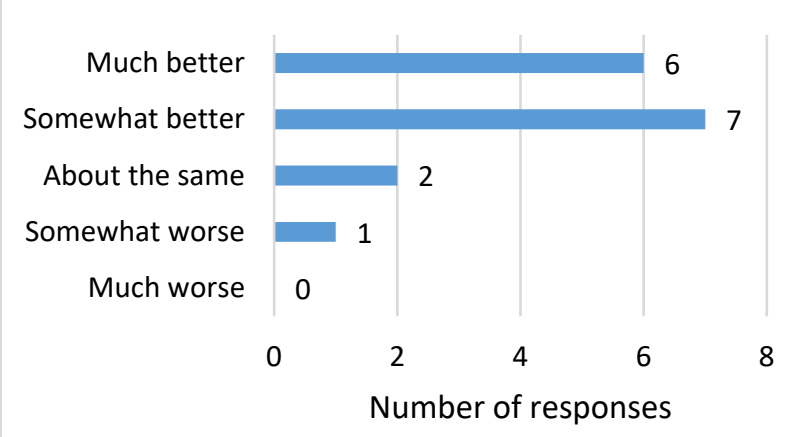

Figure 13. Student assessment of Microsoft Teams compared to alternative tools they have used $(n=16)$.

The majority of students found MS Teams to be more effective than other tools they have used. Students appreciated that MS Teams supports a variety of collaboration modes (e.g. messaging, collaborative authoring, file sharing, etc.) within a single environment, with a single login.

The project outcomes show that MS Teams helps overcome certain issues and limitations that students have encountered with other collaboration tools. The resulting project reports were of exceptional quality, and the collaborative process was visible to the course instructor. Through MS Teams, the instructor was able to monitor the projects throughout their evolution and provide formative feedback throughout the project development phase.

\section{CONCLUSIONS}

The study provides evidence that Microsoft Teams is an effective platform for collaborative knowledge building, supported by Ashby's sustainability assessment method to provide scaffolding. Students are comfortable giving and receiving constructive feedback, and they don't mind having their contributions visible to the course instructor or teaching assistant. Students rated their collaborative abilities at the end of the project to be good to excellent, and agreed that the project using Ashby's method was very effective in developing those abilities. They found MS Teams to be easy to learn and use, and more useful than other collaboration tools they have used in the past.

\section{ACKNOWLEDGEMENTS}

The authors wish to acknowledge the support of (redacted)'s Teaching Fellowship, and the Teaching Support Centre, for sponsoring and assisting with this project.

\section{REFERENCES}

[1] G. Stahl, Group Cognition: Computer Support for Building Collaborative Knowledge. Cambridge, MA: MIT Press, 2006.

[2] M. Scardamalia and C. Bereiter, "Knowledge building: Theory, pedagogy, and technology," The Cambridge Handbook of the Learning Sciences, pp. 97-115, 2006.

[3] D. P. Brandon and A. B. Hollingshead, "Collaborative learning and computer-supported groups," Communication education, vol. 48, no. 2, pp. 109-126, 1999.

[4] G. Stahl, "A model of collaborative knowledgebuilding," in Proceedings of the Fourth International Conference of the Learning Sciences, 2000, vol. 10, pp. 70-77.

[5] G. Singh, L. Hawkins, and G. Whymark, "An integrated model of collaborative knowledge building," Interdisciplinary Journal of Knowledge and Learning Objects, vol. 3, pp. 85-105, 2007.

[6] P. Resta and T. Laferrière, "Technology in support of collaborative learning," Educational Psychology Review, vol. 19, no. 1, pp. 65-83, 2007.

[7] L. Lipponen, K. Hakkarainen, and S. Paavola, "Practices and orientations of CSCL," What we know about CSCL, pp. 31-50, 2004.

[8] D. Denton, "Enhancing Instruction through Constructivism, Cooperative Learning, and Cloud Computing.," TechTrends: Linking Research \& Practice to Improve Learning, vol. 56, no. 4, pp. 34 - 41, 2012.

[9] R. Buchal and E. Songsore, "Collaborative Knowledge Building using Microsoft SharePoint," in Proc. 2018 Canadian Engineering Education Association Conf., 2018.

[10] S. K. W. Chu and M. D. M. KENNEDY, "Using online collaborative tools for groups to co-construct knowledge," Online Information Review, vol. 35, no. 4, pp. 4-4, 2011.

[11] S. Hadjerrouit, "Wiki as a collaborative writing tool in teacher education: Evaluation and suggestions for effective use," Computers in Human Behavior, vol. 32, pp. 301-312, 2014. 
[12] K. Stegmann, A. Weinberger, and F. Fischer, "Facilitating argumentative knowledge construction with computer-supported collaboration scripts," International Journal of Computer-Supported Collaborative Learning, vol. 2, no. 4, pp. 421-447, 2007.

[13] “Microsoft Teams." [Online]. Available: https://products.office.com/en-CA/microsoftteams/. [Accessed: 27-Feb-2019]

[14] M. Ashby, D. Ferrer, and J. Bruce, "Materials and Sustainable Development-a White Paper," Granta Design, Cambridge ( www. grantadesign. com/education/), 2013.

[15] M. Ashby and T. Vakhitova, "Active-Learning Took Kit - Sustainable Development," Granta Design, Cambridge ( www. grantadesign. com/education/), 2017.

[16] "What is CES Edupack." [Online]. Available: http://www.grantadesign.com/education/edupack/. [Accessed: 27-Feb-2019] 